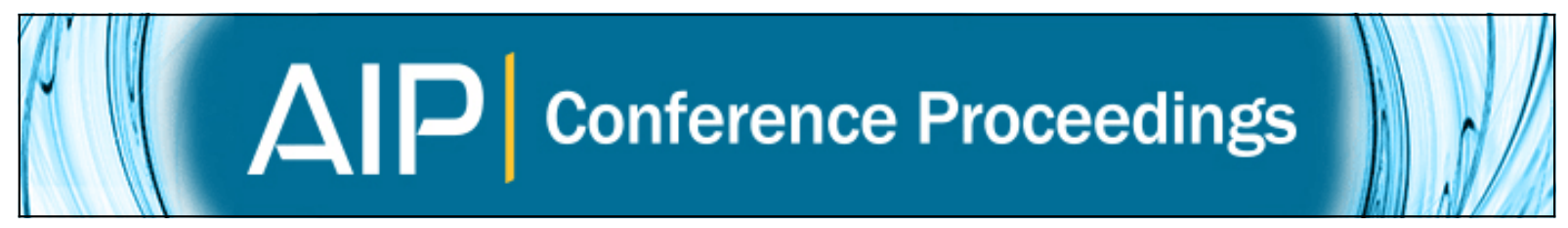

\title{
Medical image segmentation to estimate HER2 gene status in breast cancer
}

Guillermo Palacios-Navarro, José Manuel Acirón-Pomar, Enrique Vilchez-Sorribas, and Eddie Galarza

Zambrano

Citation: AIP Conference Proceedings 1705, 020026 (2016); doi: 10.1063/1.4940274

View online: http://dx.doi.org/10.1063/1.4940274

View Table of Contents: http://scitation.aip.org/content/aip/proceeding/aipcp/1705?ver=pdfcov

Published by the AIP Publishing

\section{Articles you may be interested in}

Automated fibroglandular tissue segmentation and volumetric density estimation in breast MRI using an atlasaided fuzzy C-means method

Med. Phys. 40, 122302 (2013); 10.1118/1.4829496

Automated chest wall line detection for whole-breast segmentation in sagittal breast MR images

Med. Phys. 40, 042301 (2013); 10.1118/1.4793255

Estimation of breast percent density in raw and processed full field digital mammography images via adaptive fuzzy c-means clustering and support vector machine segmentation

Med. Phys. 39, 4903 (2012); 10.1118/1.4736530

Digital Processing and Segmentation of Breast Microcalcifications Images Obtained by a Si Microstrips Detector:

Preliminary Results

AIP Conf. Proc. 885, 198 (2007); 10.1063/1.2563194

Computerized image analysis: Estimation of breast density on mammograms

Med. Phys. 28, 1056 (2001); 10.1118/1.1376640 


\title{
Medical Image Segmentation to Estimate HER2 Gene Status in Breast Cancer
}

\author{
Guillermo Palacios-Navarro ${ }^{1,2,3, \text { a) }}$, José Manuel Acirón-Pomar' ${ }^{1}$, Enrique Vilchez- \\ Sorribas ${ }^{1}$ and Eddie Galarza Zambrano ${ }^{2, \text { b) }}$
}

\author{
1Dept. Ingeniería Eléctrónica y Comunicaciones. Universidad de Zaragoza. Teruel, Spain \\ ${ }^{2}$ Dept. Eléctrica y Electrónica. Universidad de las Fuerzas Armadas ESPE, Sangolquí, Ecuador. \\ ${ }^{3}$ Prometeo Project Researcher (SENESCYT), Ecuador. \\ a)guillermo.palacios@unizar.es \\ b) eegalarza@espe.edu.ec
}

\begin{abstract}
This work deals with the estimation of HER2 Gene status in breast tumour images treated with in situ hybridization techniques (ISH). We propose a simple algorithm to obtain the amplification factor of HER2 gene. The obtained results are very close to those obtained by specialists in a manual way. The developed algorithm is based on colour image segmentation and has been included in a software application tool for breast tumour analysis. The developed tool focus on the estimation of the seriousness of tumours, facilitating the work of pathologists and contributing to a better diagnosis.
\end{abstract}

\section{INTRODUCTION}

Breast cancer is the most frequently diagnosed cancer and a leading cause of cancer deaths in women, being a serious threat to the health of the population [1]. Although screening programs and advances in the field of adjuvant systemic treatment decreased overall breast cancer mortality, a significant number of patients will eventually develop metastatic disease with a severe decrease in quality of life [2]. Breast cancer is a heterogeneous disease consisting of various subtypes with major disparities in terms of pathobiology, clinical course and prognosis [3].

One of the most common breast cancer subtypes which are usually distinguished in routine clinically practice is HER2-positive. The HER2-positive subtype is characterized by the over expression and/or amplification of HER2, a transmembrane tyrosine kinase receptor of the family of human growth-factor receptors [4]. Patients with HER2positive breast cancer derive significant benefit from treatment with HER2 targeted therapies such as the monoclonal antibodies trastuzumab, pertuzumab and T-DM1 or the tyrosine-kinase inhibitor lapatinib [5].

HER2 positive breast cancer has a high propensity for brain metastases, a devastating and challenging complication with only limited treatment options [6]. Recently, the heterodimers of HER2 and HER3 were identified as the most potent inducers of growth-factor signal transduction in HER2-positive breast cancer [7]. Furthermore, the co-expression of HER2 and HER3 was shown to induce migration and increase invasiveness of breast cancer cells [8].

Breast cancers with HER2 alterations are critical to identify because such tumours require unique treatment, including the use of targeted therapies. HER2 alterations at the DNA (amplification) and protein (over expression) level usually occur in concert, and both in situ hybridization and immunohistochemistry can be accurate methods to assess these alterations. The determination of HER2 influences the clinical decision-taking at a prognosis level. The 
HER2 status, together with other factors, can affect the decision of giving, or not, adjuvant therapy in women with breast cancer. In addition, the information about the status of HER2 is crucial to decide the use of therapy antiHER2.

Numerous studies have established that the trastuzumab anti-HER2 monoclonal antibody has a great efficiency in the treatment of all the stages of breast cancer [9]. Patients with HER2 positive metastatic breast cancer, trastuzumab administered with chemotherapy increases the rate of answers, the progression, free interval and the survival, being also active in monotherapy. It is very remarkable that the use of adjuvant trastuzumab (post operatory) in patients with early HER2 positive breast cancer reduces to the half the risk of relapses and reduces in a third the risk of mortality [10].

The development of computer-aided detection (CAD) systems based on image segmentation has shown its efficacy to improve diagnostic accuracy in breast cancer and therapeutic planning [11][12][13][14]. Automatic segmentation has the potential to positively impact clinical medicine by freeing specialists from the burden of manual labeling and by providing more robust measures to aid in diagnosis of diseases. In situ hybridization techniques are very useful to quantitatively detect amplification of this gene [15]. The main goal of this paper deals with the estimation of the HER2 gene amplification in an automatic way, facilitating valuable information for clinical decision-making at a response prediction level.

This paper is organized as follows. Section 2 introduces the HER2 gene status together with the in situ hybridization techniques. Section 3 deals with the developed algorithm to determine the HER2 amplification factor. Section 4 shows some results obtained for Silver In Situ Hybridization (SISH) images and section 5 presents the conclusions and the future work.

\section{HER2 STATUS IN SISH IMAGES}

Determination of HER2 status in breast cancer is very important in the diagnosis of breast cancer by pathologists [16][17][18]. The accurate detection of the HER2 gene amplification is of vital importance as the adjuvant or neoadjuvant therapy decisions rely on the scores that are given by these tests [19].

Different test assays for the HER2 status assessment include immunohistochemistry (ICH) and/or in situ hybridization (ISH) techniques with different methods as fluorescence (FISH), silver (SISH) or chromogenic (CISH) labelled probes [17][19].

Hybridization techniques are normally used in the diagnosis of diseases, identification of pathogenic microorganisms, study of profiles of gene expression, the location of genes in chromosomes or RNA in tissues (hybridization in situ) as well as in the comparison of species carrying out the hybridization of DNA.

In situ Hybridization (ISH) is one of the most used techniques, which is based on the ability that nucleic acids for self-hybridization (existing ADN or ARN sequences complementary with another one). This technique is very useful, for instance, to identify the nucleotides sequence, in certain genetic diseases.

Amplification of the HER2 gene occurs in approximately 15 to 25 percent of breast cancers, and is associated with aggressive tumour behaviour [20]. In many clinical studies, amplification of HER2 has been shown to be associated with a poor clinical outcome for women with invasive breast cancer and correlated with several negative prognostic variables such us oestrogen receptor (ER) negative status. HER2 gene status is reported as a function of the ratio of the average number of copies of the HER2 gene to the average number of copies of Chromosome 17 (Chr17) per cell in an invasive breast carcinoma. HER2 gene status is classified using the following guidelines [21]. A sample is negative for HER2 gene amplification if the HER2/Chr17 ratio is less than 1.8. A sample is equivocal for HER2 gene amplification if the HER2/Chr17 Identification of Appropriate Staining Pattern ratio is either equal to or falls between 1.8 and 2.2. Finally, if the HER2/Chr17 ratio is greater than 2.2, a sample is positive for HER2 gene amplification. This classification is summarized in Table 1. 


\section{Table 1}

TABLE 1. Her2 testing reported results

\begin{tabular}{|c|c|}
\hline Average HER2/Chr17 ratio & Reported Result \\
\hline HER2/Chr17 < 1.8 & $\begin{array}{c}\text { Negative for HER2 gene } \\
\text { amplification }\end{array}$ \\
\hline $1.8 \leq \mathrm{HER} 2 / \mathrm{Chr} 17 \leq 2.2$ & $\begin{array}{c}\text { Equivocal for HER2 gene } \\
\text { amplification }\end{array}$ \\
\hline HER2/Chr17 > 2.2 & $\begin{array}{c}\text { Positive for HER2 gene } \\
\text { amplification }\end{array}$ \\
\hline
\end{tabular}

The probes are visualized using silver in situ hybridization (SISH) and appear as discrete black dots in the nuclei of normal cells (serving as internal positive controls for staining) and in invasive carcinoma. The chromosomes appear as red dots (see fig. 1a). This strategy allows HER2 gene status to be determined in the context of its chromosomal state, using standard light microscopy with $20 \mathrm{X}, 40 \mathrm{X}$, and/or 60x objectives. SISH signals are visualized as single copies, multiple copies and clusters (see fig. 1b).

\section{Figure 1}

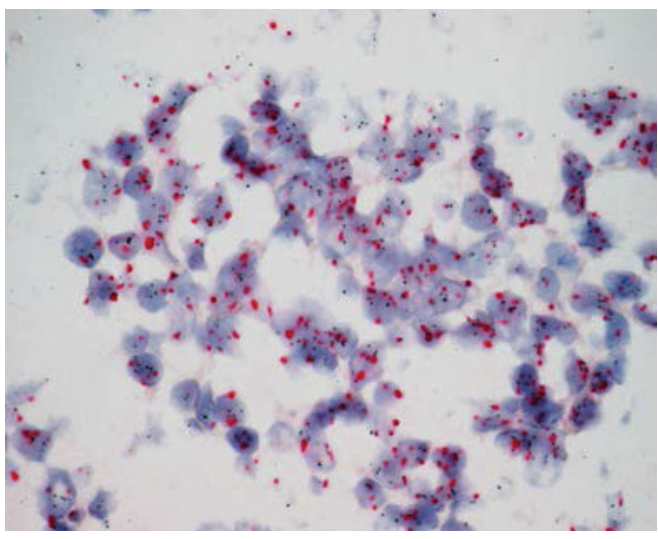

(a)

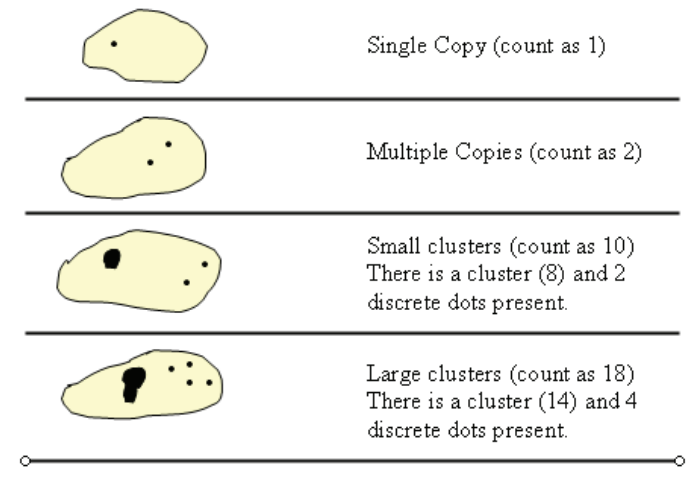

(b)

FIGURE 1. a) SISH image with chromosomes in red colour and genes in black colour. b) Signal Enumeration for HER2 gene.

Figure $1 \mathrm{~b}$ reflects the great variety of HER2 staining patterns we can find after the probe staining process. Note that the number of clusters as well as their density is different, reflecting tumour mutations.

One of the current problems working with these techniques deals with the interpretation of the obtained results. Interpretation errors decrease to an acceptable level when pathologists have enough experience in such techniques. Normally they work on a manual basis, so results are individual-dependent and for this reason it is necessary to create an automatic process to increase accuracy. 


\section{PROPOSED ALGORITHM}

\section{A. Stage 1. Gene Size Selection}

The user (pathologist) selects an arbitrary number of genes which will be used to calculate the gene size (average value) for that particular image. The program automatically calculates the appropriate size in pixels for a gene (average). This process should be done for every single image to ensure the accuracy of the estimation. The gene size value is stored and it will be used in the current session. Figures $2 \mathrm{a}$ and $2 \mathrm{~b}$ show the estimation procedure.

\section{B. Stage 2. Image Segmentation}

The RGB input image is segmented into three different clusters that correspond to the chromosomes (pink colour), genes (black colour), and cells (blue colour), respectively. The selected algorithm for this segmentation process was the k-means algorithm. Previously, the image was converted into the Lab colour space. The k-means algorithm was selected for easiness and effectiveness. Figure 3 show the results of the k-means algorithm.

\section{Figure 2}

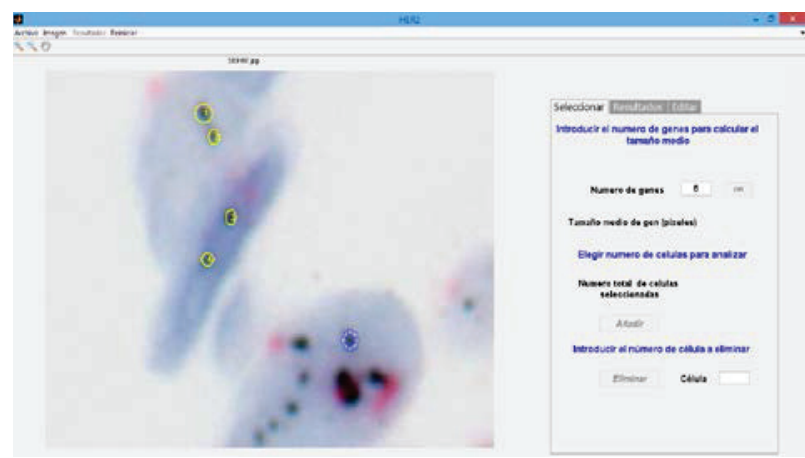

(a)

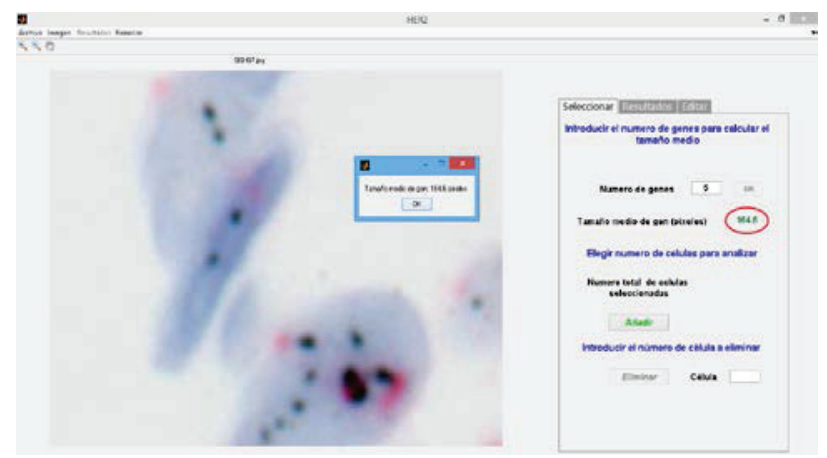

(b)

FIGURE 2. a) Screenshot showing the procedure of gene selection for size gene estimate. b) Screenshot showing the gene mean value estimate.

\section{Figure 3}

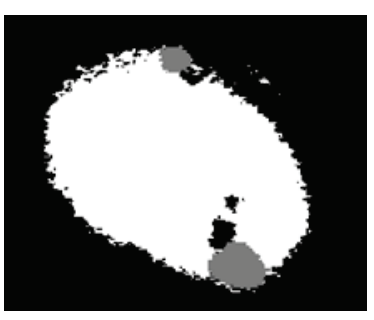

(a)

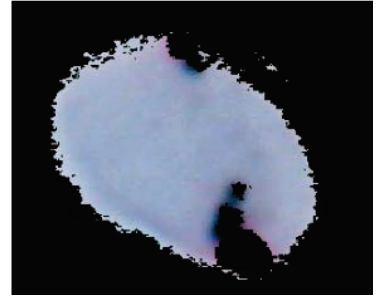

(b)

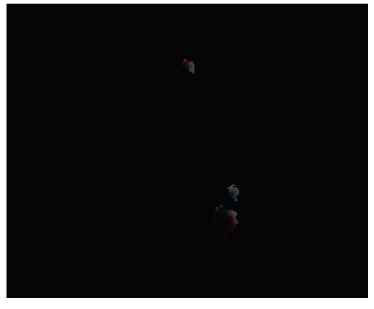

(c)

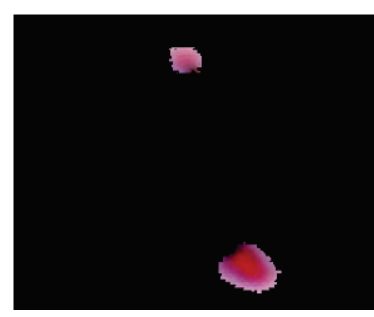

(d)

FIGURE 3. a) K-means output image with three clusters. b) objects in cluster 1 (cell). c) objects in cluster 2 (genes). d) objects in cluster 3 (chromosomes). 


\section{Stage 3. Image Dilation}

The aim at this stage deals with the dilation of the genes and chromosomes images to adjust their sizes according to their original size and shape. Some of the pixels have been lost in the former segmentation process. The procedure has been done for both genes and chromosomes images and consists of the following steps: 1) The gene/chromosome image is converted into a gray-level image, 2) Edge detection algorithm (Canny) to detect objects, 3) Image dilation, 4) Image hole-filling, 5) Image border removing, and 6) Image erosion.

\section{Stage 4. Average HER2/Chr17 Ratio}

To estimate the average HER2/Chr17 ratio we have to count the number of genes as well as the number of chromosomes exhibited in the cell. The abovementioned ratio is the quotient of the two magnitudes.

Chromosome counting process: the proposed chromosome counting process is very simple, which is based in a $3 \times 3$ pixel neighbourhood analysis. Pixels belonging to an object and their adjacent pixels are grouped into regions and labelled subsequently. The number of different regions will be the total number of objects. Firstly, the image has been converted to a black and white image. A threshold can be established in order to discard regions having a small number of pixels (as non-meaningful regions) to preserve an appropriate number of chromosomes. The threshold has been established in 20 pixels.

Gene counting process: The number of genes in the selected cell is calculated as the ratio of the total gene area and the gene size mean value (previously stored by the user).

\section{RESULTS}

Figures $4 \mathrm{a}$ and $4 \mathrm{~b}$ show the obtained amplification factor (AF) and the gene Status (GS) according to the classification made in table 1 in section 2. Figure 4 a belongs to a breast carcinoma which is negative for HER2 gene amplification, whereas figure $4 \mathrm{~b}$ belongs to a breast carcinoma which is positive for HER2 gene amplification. Note that in both figures, there are a couple of equivocal cases.

\section{Figure 4}

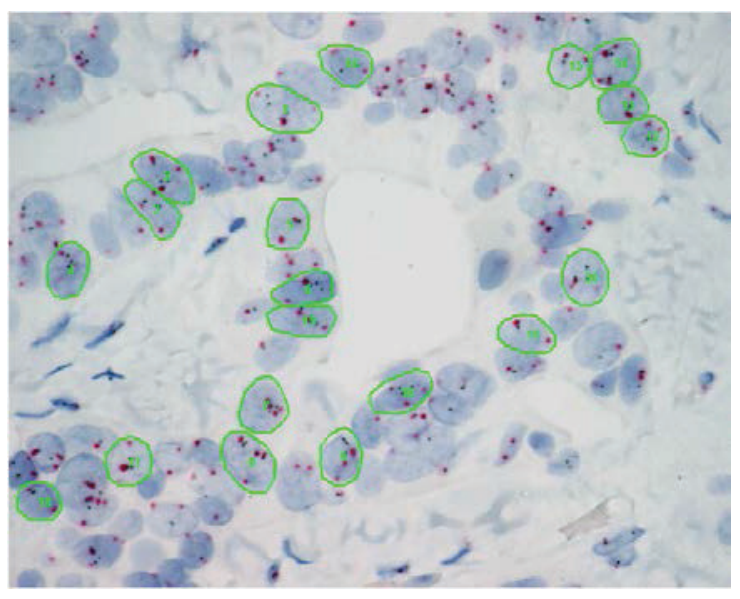

(a)

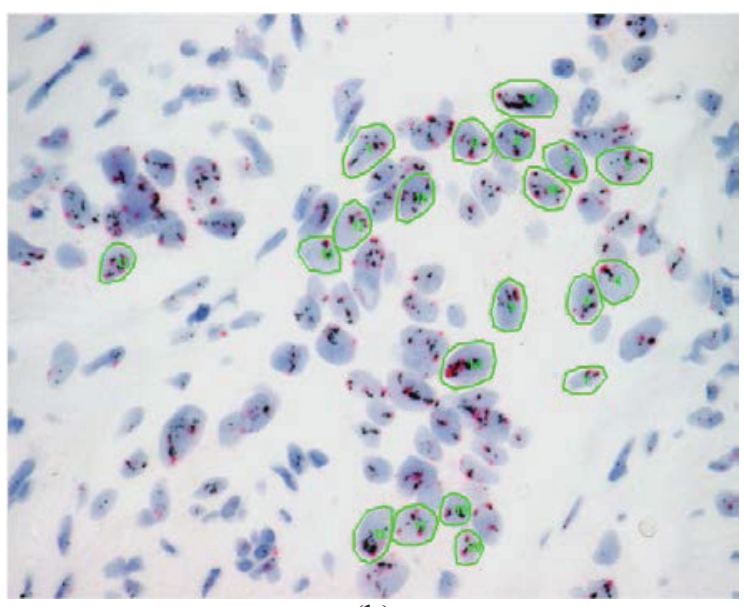

(b)

FIGURE 4. Original images treated with a Silver In Situ Hybridization together with the selected images to be analyzed. a) breast carcinoma negative for HER2 gene amplification. b) breast carcinoma positive for HER2 gene amplification 


\section{Table 2}

TABLE 2. Her2 gene status results for figure 6 a after applying the algorithm to the 20 selected cells. A.F: amplification factor. G.S: gene status.

\begin{tabular}{c|ccc|c}
\hline Cell & Number of Genes & Number of Chromosomes & A.F. & G. S. \\
\hline 1 & 3,1 & 3 & 1,1 & NO \\
2 & 6,5 & 3 & 2,18 & EQUIVOCAL \\
3 & 2,5 & 3 & 0,83 & NO \\
4 & 2,1 & 8 & 0,26 & NO \\
5 & 2 & 2 & 1,01 & NO \\
6 & 2,4 & 2 & 1,23 & NO \\
7 & 2,4 & 2 & 1,20 & NO \\
8 & 3,3 & 3 & 1,12 & NO \\
9 & 3,5 & 3 & 1,19 & NO \\
10 & 2,2 & 3 & 0,73 & NO \\
11 & 1,1 & 2 & 0,56 & NO \\
12 & 2,2 & 3 & 0,73 & NO \\
13 & 1,6 & 2 & 0,84 & NO \\
14 & 3,5 & 4 & 0,88 & NO \\
15 & 1,2 & 2 & 0,62 & NO \\
16 & 2 & 3 & 0,66 & NO \\
17 & 3,8 & 2 & 1,90 & EQUIVOCAL \\
18 & 2,2 & 3 & 0,75 & NO \\
19 & 1,1 & 3 & 0,38 & NO \\
20 & 3,1 & 2 & 1,54 & NO \\
\hline
\end{tabular}

Table 3

TABLE 3. Her2 gene status results for figure $6 \mathrm{~b}$ after applying the algorithm to the 20 selected cells. A.F: amplification factor. G.S: gene status.

\begin{tabular}{c|ccc|c}
\hline Cell & Number of Genes & Number of Chromosomes & A.F. & G. S. \\
\hline 1 & 14,1 & 4 & 3,53 & YES \\
2 & 6,5 & 2 & 3,25 & YES \\
3 & 13,9 & 4 & 3,48 & YES \\
4 & 8,7 & 2 & 4,34 & YES \\
5 & 13,1 & 2 & 6,57 & YES \\
6 & 11,3 & 5 & 2,26 & YES \\
7 & 4,6 & 1 & 4,64 & YES \\
8 & 15,9 & 4 & 3,98 & YES \\
9 & 12,3 & 4 & 3,08 & YES \\
10 & 14,9 & 8 & 1,87 & EQUIVOCAL \\
11 & 2,2 & 6 & 3,67 & YES \\
12 & 8,2 & 3 & 2,74 & YES \\
13 & 6,4 & 2 & 3,22 & YES \\
14 & 18,8 & 5 & 3,76 & YES \\
15 & 11,7 & 4 & 2,93 & YES \\
16 & 20,3 & 4 & 5,08 & YES \\
17 & 8,2 & 3 & 2,75 & YES \\
18 & 24,5 & 4 & 6,14 & YES \\
19 & 11,1 & 2 & 5,51 & YES \\
20 & 6,15 & 3 & 2,05 & EQUIVOCAL \\
\hline
\end{tabular}




\section{CONCLUSIONS}

In this work, we have developed a tool that brings together different image segmentation techniques to solve a very common problem in the field of pathology. The target images come from the application of in situ hybridization techniques. The tool is intended for use as an adjunct to existing clinical and pathological information currently used for estimating prognosis in patients with breast cancer. We have tested the application over a wide range of SISH images, obtaining very satisfactory results. Therefore, it provides a valid tool for pathologists in the field of prevention of infectious diseases and guiding them to a more straightforward diagnosis.

Future work deals with the development of new applications to estimate tumoral parameters together with the application of these techniques to other types of tumours. The software application, as well as the processing algorithms have been developed using Matlab ${ }^{\circledR}$.

\section{ACKNOWLEDGMENTS}

Authors gratefully acknowledge the support of Prometeo Project, Secretaría de Educación Superior, Ciencia, Tecnología e Innovación from Ecuador, as well as the Fundación Antonio Gargallo and Obra Social Ibercaja for its support under the grant 2014/B0017. Authors also thank their clinical partners from the Pathological Anatomy department, at Obispo Polanco Hospital in Teruel, for their support and valuable contribution.

\section{REFERENCES}

1. K. McPherson, C.M. Steel and J.M. Dixon, "Breast cancer-epidemiology, risk factors, and genetics," BMJ 321, 624-628 (2000).

2. D.A. Berry, K.A. Cronin, S.K. Plevritis, D.G. Fryback, L. Clarke, M. Zelen, et al., "Effect of screening and adjuvant therapy on mortality from breast cancer," New Engl J Med, 353(17):1784e92 (2005).

3. C.M. Perou, T. Sorlie, M.B. Eisen, M. van de Rijn, S.S. Jeffrey, C.A. Rees, et al., "Molecular portraits of human breast tumours," Nature, 406(6797):747e52 (2000).

4. D.J. Slamon, G.M. Clark, S.G. Wong, W.J. Levin, A. Ullrich, W.L. McGuire, "Human breast cancer: correlation of relapse and survival with amplification of the HER-2/neu oncogene," Science 235(4785):177e82 (1987).

5. C.E. Geyer, J. Forster, D. Lindquist, S. Chan, C.G. Romieu, T. Pienkowski, et al., "Lapatinib plus capecitabine for HER2-positive advanced breast cancer," New Engl J Med, 355(26):2733e43 (2006).

6. N.U. Lin, C. Thomssen, F. Cardoso, D. Cameron, T. Cufer, L. Fallowfield, et al., "International guidelines for management of metastatic breast cancer (MBC) from the European School of Oncology (ESO)-MBC task force: surveillance, staging, and evaluation of patients with early-stage and metastatic breast cancer," Breast 22(3):203e10 (2013).

7. A. Zaczek, B. Brandt, K.P. Bielawski, "The diverse signaling network of EGFR, HER2, HER3 and HER4 tyrosine kinase receptors and the consequences for therapeutic approaches," Histol Histopathol 20(3):1005e15 (2015).

8. N. Aceto, S. Duss, G. Macdonald, D.S. Meyer, T.C. Roloff, N.E. Hynes, et al., "Coexpression of HER2 and HER3 receptor tyrosine kinases enhances invasion of breast cells via stimulation of interleukin-8 autocrine secretion," Breast Cancer Res BCR 14(5):R131 (2012).

9. A.C. Wolff, et al., American Society of Clinical Oncology/College of American Pathologists. "Guideline recommendations for human epidermal growth factor receptor 2 testing in breast cancer," J Clin Oncol 25, $118-45$ (2007).

10. M. Untch, et al., "Estimating the magnitude of trastuzumab effects within patient subgroups in the HERA trial," Ann Oncol 19,1090-6 (2008).

11. G. Palacios, J.R. Beltran, "Cell nuclei segmentation combining multiresolution analysis, clustering methods and colour spaces," Proceedings of the IEEE International Conference Machine Vision and Image Processing. Kildare, Ireland, 2007, pp. 91-97. 
12. G. Palacios, J. R. Beltran, "A wavelet transform based multiresolution edge detection and classification schema," Proceedings of SPIE 7000, Optical and Digital Image Processing, 70000U, Strasbourg, France, 2008.

13. G. Palacios, R. Lacuesta, P. Ramos, "Automatic estrogens detection in breast tumour images treated with immune-histochemical techniques," Proceedings of the International Conference on Image Processing, Computer Vision, and Pattern Recognition, Las Vegas, NV, United States, 2010, pp. 87-92.

14. G. Palacios-Navarro, "Computer-aided tool for breast tumour analysis purposes," Proceedings of the International Conferences Interfaces and Human Computer Interaction, Game and Entertainment Technologies and Computer Graphics, Visualization, Computer Vision and Image Processing. Las Palmas de Gran Canaria, España, 2104, pp. 281-285.

15. M. Bilous et al., "Current perspectives on HER2 Testing: A review of national testing guidelines," Mod Pathol., 173-182 (2003).

16. W.M.Hanna, J. Ruschoff, M. Bilous, R.A. Coudry, M. Dowsett, et al., "HER2 in situ hybridization in breast cancer: clinical implications of polysomy 17 and genetic heterogeneity," Mod Pathol (2013).

17. J. Jacquemier, F. Spyratos, B. Esterni, M.J. Mozziconacci, M. Antoine, et al., "SISH/CISH or qPCR as alternative techniques to FISH for determination of HER2 amplification status on breast tumors core needle biopsies: a multicenter experience based on 840 cases," BMC Cancer 13: 351 (2013).

18. A. Lebeau, A. Turzynski, S. Braun, W. Behrhof, B. Fleige, et al., "Reliability of human epidermal growth factor receptor 2 immunohistochemistry in breast core needle biopsies," J Clin Oncol 28, 3264-3270, 2010.

19. A.C. Wolff, M.E. Hammond, D.G. Hicks, M. Dowsett, L.M. McShane, et al., "Recommendations for Human Epidermal Growth Factor Receptor 2 Testing in Breast Cancer: American Society of Clinical Oncology/College of American Pathologists Clinical Practice Guideline Update," J Clin Oncol., (2013).

20. D. J. Slamon, W. Godolphin, L. A. Jones et al., "Studies of the HER-2/neu proto-ongene in human breast and ovarian cancer," Science, 244, 707-712 (1989).

21. A.C. Wolff, M.E.H. Hammond, J.N. Schwartz et al., American Society of Clinical Oncology/College of American Pathologists "Guideline Recommendations for Human Epidermal Growth Factor Receptor 2 Testing in Breast Cancer," Arch Pathol Lab Med, 131,18-43 (2007). 